\title{
Microsurgical reconstruction of combined tracheal and total esophageal defects
}

\author{
Shadi Ghali, MD, ${ }^{\mathrm{a}}$ Edward I. Chang, MD, ${ }^{\mathrm{a}}$ David C. Rice, MD, ${ }^{\mathrm{b}}$ Garrett L. Walsh, MD, ${ }^{\mathrm{b}}$ and \\ Peirong Yu, MD
}

Objective: Lesions involving both the trachea and the esophagus are often considered inoperable because of the lack of reliable reconstruction. The purpose of this study was to review our experience of combined supercharged jejunal and other flaps for tracheal and esophageal reconstruction.

Methods: A retrospective review of 5 consecutive cases with combined tracheal and total esophageal defects was performed. The esophageal defect was reconstructed with a supercharged jejunal flap, and the trachea was reconstructed with a free anterolateral thigh flap or a pedicled muscle flap.

Results: Primary diagnosis included tracheostoma recurrence after a total laryngectomy for laryngeal cancer in 2 patients and tracheoesophageal fistula due to esophageal stenting for complications from prior treatment for non-Hodgkin's lymphoma, parathyroid cancer, and esophageal cancer in 3 patients, respectively. Tracheal and esophageal reconstructions were staged in 4 patients, and 1 patient received simultaneous reconstruction. Tracheal necrosis developed in 1 patient with a mediastinal tracheostoma, and the patient eventually died of infection 2 months later. The other 4 patients recovered well and resumed an oral diet.

Conclusions: Complex and often life-threatening lesions involving both the trachea and the esophagus are not necessarily inoperable. With careful planning, these combined defects can be safely reconstructed with multiple flaps with good functional outcomes and reasonable survival. (J Thorac Cardiovasc Surg 2015;150:1261-6)

See related commentary on pages $1266-7$.

Esophageal reconstruction after an esophagectomy is commonly accomplished with a gastric pull-up procedure. ${ }^{1}$ When the stomach is unavailable, previously radiated, or unable to reach the pharynx or when a concomitant total laryngopharyngectomy is performed, a colon interposition or a supercharged jejunal flap is a common alternative..$^{2-4}$ Although the supercharged jejunal flap is a complex and lengthy procedure requiring microsurgical skills, it provides a well-vascularized conduit because of the additional set of vascular anastomosis in the neck. When the trachea is involved in the disease in addition to a total esophageal defect due to cancer or tracheoesophageal fistula, reconstruction of both organs can be extremely challenging, if not impossible. With significant loss of the cervical trachea, formation of an anterior mediastinal

\footnotetext{
From the Departments of ${ }^{\mathrm{a} P l a s t i c}$ Surgery and ${ }^{\mathrm{b}}$ Thoracic and Cardiovascular Surgery, the University of Texas MD Anderson Cancer Center, Houston, Tex.

Received for publication June 22, 2011; revisions received Sept 8, 2011; accepted for publication Oct 9, 2011; available ahead of print May 18, 2015.

Address for reprints: Peirong Yu, MD, Department of Plastic Surgery, Unit 1488, 1515 Holcombe Blvd, Houston, TX 77030 (E-mail: peirongyu @ mdanderson.org). $0022-5223 / \$ 36.00$

Copyright (c) 2015 by The American Association for Thoracic Surgery http://dx.doi.org/10.1016/j.jtcvs.2011.10.100
}

tracheostomy may provide an immediate airway but carries high morbidity and mortality rates. ${ }^{5}$ Defects in the lower trachea near the carina due to a tracheoesophageal fistula can be life-threatening and difficult to repair. The goals of reconstruction for these combined tracheal and esophageal defects are to provide a secure airway, reliable coverage of major blood vessels, and restoration of the continuity of the alimentary tract. When possible, these goals can be achieved in a single stage, but the enormity and complexity of these procedures frequently require staged reconstructions, especially in reestablishing alimentary tract continuity. In this series, we present a single reconstructive surgeon's experience in the reconstruction of combined tracheal, esophageal, and neck defects using a combination of a supercharged jejunum and other pedicled or free flaps.

\section{PATIENTS AND METHODS}

From November 2005 to July 2010, 5 male patients ranging in age from 38 to 76 years presented with lesions involving both the airway and the esophagus requiring resection of the trachea and a total esophagectomy. This retrospective review was approved by the institutional review board of the University of Texas, MD Anderson Cancer Center.

Primary diagnosis included tracheostoma recurrence after a total laryngectomy for laryngeal cancer in 2 patients and tracheoesophageal fistula due to esophageal stenting for complications from prior treatment for non-Hodgkin's lymphoma, parathyroid cancer, and esophageal cancer in 3 patients, respectively. Reconstruction of the esophagus was achieved using a supercharged jejunal flap in all patients. Reconstruction of the tracheal/bronchial defects was accomplished with a free anterolateral thigh (ALT) flap in 2 patients, a pedicled pectoralis major muscle flap in 1 patient, a pedicled latissimus dorsi muscle flap in 1 patient, and an intercostal muscle flap in 1 patient (Table 1). 


\section{Abbreviation and Acronym \\ $\mathrm{ALT}=$ anterolateral thigh}

\section{Surgical Techniques}

Supercharged jejunal flap. The supercharged jejunal flap was prepared as previously described. ${ }^{6,7}$ Briefly, the mesentery of the proximal and mid-jejunum was closely examined using a fiberoptic light to transilluminate the tissues and elucidate the vascular anatomy. The first mesenteric branch beyond the ligament of Treitz was identified and preserved. The second mesenteric branch was dissected down to the level of the superior mesenteric artery and vein, and ultimately divided to serve as the vascular pedicle for supercharging the proximal flap. The third mesenteric branch was ligated and divided. The mesentery between the second and third branches was divided up to the serosal border, allowing the jejunal segment to unfurl (Figure 1). Secondary arcade vessels between the third and fourth mesenteric branches were preserved so that the segment of bowel normally supplied by the third branch could be now supplied by the pedicled fourth branch through intact arcade vessels. The jejunum between the first and second mesentery vessels was divided with a linear cutting stapler.

There are 2 potential routes for transferring the jejunal flap to the neck. For immediate reconstruction, the retrocardiac (orthotopic) route was usually chosen. For delayed reconstruction, the substernal route was chosen and a portion of the manubrium, clavicular head, and first rib was removed to enlarge the thoracic inlet and avoid constriction on the jejunal conduit. The proximal jejunum was then revascularized using microsurgical techniques. Any excess length of jejunal conduit was removed to minimize redundancy in the neck. The proximal 3 to $5 \mathrm{~cm}$ of jejunum based on 1 or 2 terminal arcade vessels was exteriorized as a postoperative monitoring segment, which was usually removed at bedside 7 to 10 days after surgery. The esophagojejunal anastomosis was then performed in the neck. Intestinal continuity was reestablished in the abdomen through a gastrojejunal anastomosis using the posterior wall of the stomach or through a Roux-en-Y jejunojejunal anastomosis if a gastrectomy was performed. A feeding jejunostomy tube was routinely placed.

Anterolateral thigh free flap. Flap design was outlined on the basis of the $\mathrm{ABC}$ perforator system previously described by $\mathrm{Yu}^{8}$ and $\mathrm{Yu}$ and colleagues. ${ }^{9}$ Briefly, a straight line (AP line) was drawn connecting the anterior superior iliac spine and the superolateral corner of the patella, and its midpoint was marked. Perforator B was marked at the midpoint and $1.5 \mathrm{~cm}$ lateral to the AP line. Perforators $\mathrm{A}$ and $\mathrm{C}$ were marked $5 \mathrm{~cm}$ proximal and distal to perforator B, respectively. A flap width of $8 \mathrm{~cm}$ is usually required for circumferential tracheal reconstruction to obtain a luminal diameter of $2.5 \mathrm{~cm}$. The flap design was centered on perforator $\mathrm{B}$. The medial incision was made first, and the dissection was carried out in a subfascial plane until the cutaneous perforators were identified. The intermuscular space between the vastus lateralis and the rectus femoris was then entered, and the descending branch of the lateral circumflex femoris artery was isolated. Various amounts of the vastus lateralis muscle also can be included with the flap. The flap was then harvested by dividing the vascular pedicle. For circumferential tracheal reconstruction, the flap was tubed and 1 end of the tube was anastomosed to the tracheal end. The other end was brought out to the neck or chest and sutured to the surrounding skin to mature the stoma. Vascular anastomosis of the flap pedicle to recipient vessels, usually the transverse cervical vessels or internal thoracic vessels, was then performed under an operating microscope. An endotracheal tube was placed in the tubed flap just beyond the anastomosis with the native trachea under bronchoscopic guide.

Case 1. A 76-year-old man with a history of laryngeal squamous cell carcinoma was treated 8 years previously with chemoradiation and neck dissections. Tracheal stenosis developed 4 years later, and a permanent tracheostomy was performed. He then presented to the University of Texas MD Anderson Cancer Center with a moderately differentiated squamous cell carcinoma of the esophagus involving the cervical tracheostomy. Total laryngectomy, tracheal resection, and a total esophagectomy were performed. A mediastinal tracheostoma was created by resecting the manubrium, the medial parts of bilateral first and second ribs, and both clavicular heads and advancing the chest skin to the remaining $5 \mathrm{~cm}$ of trachea. An initial attempt for gastric conduit reconstruction was abandoned because of inadequate length. Therefore, the supercharged jejunal flap was used instead via the retrocardiac route with the left internal thoracic vessels as recipient vessels. An omental flap was placed between the trachea and the innominate artery. A right pectoralis major muscle flap was also used to further protect the trachea and major blood vessels (Figure 2). Skin grafts were placed over the muscle flap to address skin shortage in the neck and upper chest. Necrosis of the remaining trachea with exposure of the aorta then developed in the patient. This was debrided down to within $1 \mathrm{~cm}$ of the carina. Circumferential tracheal reconstruction was accomplished with a free ALT flap with vascular anastomoses to the right internal thoracic vessels (Figure 3). A Y-type stent was placed through the reconstructed trachea down to the left and right main stem bronchi. Flexible bronchoscopy 2 weeks later confirmed full healing of the flap to the carina, and he was breathing spontaneously. The patient contracted a multidrug-resistant pseudomonas infection resulting in multiorgan failure, and he died 3 weeks later, despite viability of the flap for tracheal reconstruction.

Case 2. A 74-year-old man was initially treated 15 years earlier for laryngeal squamous cell carcinoma with radiotherapy alone. One year later, a recurrence developed and he underwent a salvage total laryngectomy. $\mathrm{He}$ was disease-free for 14 years and then presented with dysphagia and was found to have a stomal recurrence invading the esophagus. An esophagectomy, pharyngectomy, and tracheal resection were performed. The tracheal defect involved the posterior two thirds of the trachea to within $5 \mathrm{~cm}$ of the carina. To avoid a risky mediastinal tracheostomy, reconstruction of both the trachea and the esophagus was planned. A free ALT flap with a portion of the vastus lateralis muscle was used to reconstruct the trachea, and the esophagus was reconstructed with a supercharged jejunal flap (Figure 4). The jejunum was brought to the base of tongue via the native esophageal (retrocardiac) route and revascularized to the left transverse cervical vessels. The anterior neck skin defect was resurfaced with part of the ALT flap (Figure 5), and the vastus lateralis muscle was used to protect the major blood vessels in the neck. A Bivona tube (Smiths Medical, Dublin, Ohio) was initially placed in the reconstructed trachea, which was changed to a soft Larry tube (Platon Medical Ltd, Eastbourne, England) 1 week later. $\mathrm{He}$ recovered well and was discharged 2 weeks postoperatively. He then completed adjuvant radiotherapy and continued to do well at 5 months follow-up tolerating a soft diet with supplemental tube feeds.

Case 3. A 38-year-old man was initially treated with multiple surgeries and radiotherapy since 1995 at an outside institution for a parathyroid cancer. The tumor eventually invaded the esophagus, which was treated with photodynamic therapy. An esophageal stricture then developed, which was managed with a stent. The stent eventually eroded through to the trachea causing a 4-cm wide tracheoesophageal fistula extending from the thoracic inlet to $2 \mathrm{~cm}$ above the carina with life-threatening pulmonary complications, including cardiopulmonary arrest. At the time of presentation to our hospital, his vocal cords were nonfunctional. A 2-stage surgery was planned. During the first stage, a total laryngectomy was performed, the cervical and upper thoracic esophagus was debrided, and a spit fistula was created in the neck. A pectoralis major muscle flap was raised from the left chest and split into 3 segments. The medial segment was used to protect the innominate artery. The middle segment was used to repair the posterior tracheal wall over a Bivona tube and obliterate the distal esophageal lumen, which was demucosalized with an Argon beam. The lateral segment was used to obliterate the dead space in the neck above the tracheostoma. The patient recovered well from surgery with complete healing of the tracheal defect. At the second-stage surgery 6 months later, total esophageal reconstruction was performed with a supercharged jejunal flap via a substernal route with revascularization to the transverse cervical vessels. After an unremarkable 
TABLE 1. Patient information

\begin{tabular}{|c|c|c|c|c|c|c|c|}
\hline Patient & Age, y & Primary diagnosis & Current problem & Resection and reconstruction & $\begin{array}{l}\text { Postoperative } \\
\text { complications }\end{array}$ & D/c POD & Outcome \\
\hline 1 & 76 & Laryngeal cancer & Stomal recurrence & $\begin{array}{l}\text { First: TL, TEgt, TR, MT, } \\
\text { SCJf + PMMf } \\
\text { Second: free ALT flap }\end{array}$ & $\begin{array}{r}\text { Tracheal necrosis, } \\
\text { Sepsis + MOF }\end{array}$ & $\mathrm{n} / \mathrm{a}$ & $\begin{array}{l}\text { Death } \\
2 \text { mo }\end{array}$ \\
\hline 2 & 74 & Laryngeal cancer & Stomal recurrence & TEgt-SCJf, TR-ALT & None & 14 & $\begin{array}{l}\text { NED } \\
5 \mathrm{mo}\end{array}$ \\
\hline 3 & 38 & Parathyroid carcinoma & TE fistula & $\begin{array}{l}\text { First: TL, TEgt, PMMf } \\
\text { Second: SCJf }\end{array}$ & None & $\begin{array}{l}13 \\
15\end{array}$ & $\begin{array}{c}\text { NED } \\
4 \mathrm{y}\end{array}$ \\
\hline 4 & 54 & Lymphoma & TE fistula & $\begin{array}{l}\text { First: TEgt, LD + SA } \\
\text { Second: SCJf + PMMf }\end{array}$ & $\begin{array}{c}\text { Pneumonia, AF, } \\
\text { ARDS, ileus }\end{array}$ & $\begin{array}{l}35 \\
18\end{array}$ & $\begin{array}{c}\text { DOD } \\
3 y\end{array}$ \\
\hline 5 & 53 & Esophageal cancer & TE fistula & $\begin{array}{l}\text { First: TEgt, lobectomy, ICMf } \\
\text { Second: SCJf }\end{array}$ & None & $\begin{array}{l}10 \\
12\end{array}$ & $\begin{array}{c}\text { DOD } \\
2 \mathrm{y}\end{array}$ \\
\hline
\end{tabular}

$\overline{A F}$, Atrial fibrillation; $A L T$, anterolateral thigh flap; $A R D S$, adult respiratory distress syndrome; $D O D$, dead of disease; $I C M f$, intercostal muscle flap; $L D$, latissimus dorsi flap; $M O F$, multiple organ failure; $M T$, mediastinal tracheostomy; $n / a$, not available; $N E D$, no evidence of disease; $P M M f$, pectoralis major muscle flap; $P O D$, postoperative day; $S A$, serratus anterior flap; $S C J f$, supercharged jejunal flap; $T E$, tracheoesophageal; $T E g t$, total esophagectomy; $T L$, total laryngectomy; $T R$, tracheal resection; $D / C$, discharge.

recovery, he was discharged on postoperative day 15 . He tolerated a regular diet without the need for tube feeding and within 6 months gained 20 pounds. $\mathrm{He}$ is currently progressing well 4 years after surgery with a stable airway. Case 4. A 54-year-old man was initially diagnosed with a non-Hodgkin's lymphoma (abdomen/mediastinum) in 1982 treated with chemotherapy. In 2005, he was found to have a neck mass, and biopsy confirmed a non-Hodgkin's lymphoma. He was started on chemotherapy, but a bronchoesophageal fistula and aspiration pneumonia subsequently developed in the patient. A Polyflex (Boston Scientific, Marlborough, Mass) stent was placed and a gastrostomy was performed at an outside institution, and he was transferred to us because of persistent fistulation and life-threatening pneumonia. At surgery, the stent was removed, revealing a $2 \times 4-\mathrm{cm}$ fistula between the esophagus and the left main stem bronchus near the carina. A total esophagectomy was performed with a spit fistula in the neck. A combined latissimus/serratus muscle flap was turned into the chest cavity by removing part of the third rib. The serratus muscle segment was used to fill the intraluminal left bronchial defect with the latissimus muscle covering the tracheobronchial system and obliterating the surrounding dead space. Postoperatively, excess muscle in the left bronchus was trimmed with a YAG laser without obstruction. He then received adjuvant chemotherapy and an autologous stem cell

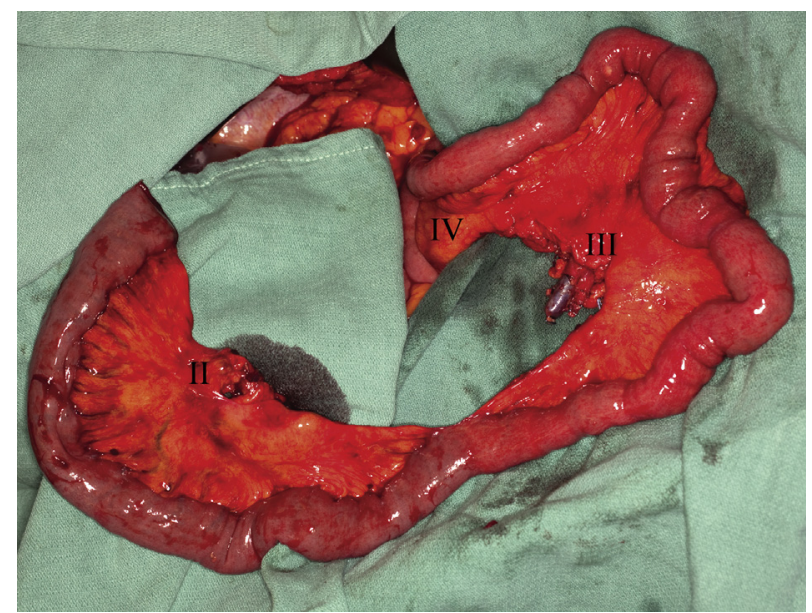

FIGURE 1. The second mesentery vessels are divided and revascularized in the neck. The third one is ligated, and the fourth one is left intact. The mesentery between the second and third vessels is divided to the bowel wall to unfurl the jejunum. transplant 6 months later. He proceeded to the second-stage esophageal reconstruction with a supercharged jejunal flap 1 year later. The jejunum was brought to the neck via the substernal route, and vascular anastomoses were performed to the internal thoracic vessels. He was discharged on postoperative day 18 . He subsequently tolerated a normal diet without supplementary tube feeds and gained weight. He lived for 3 years before dying of lymphoma.

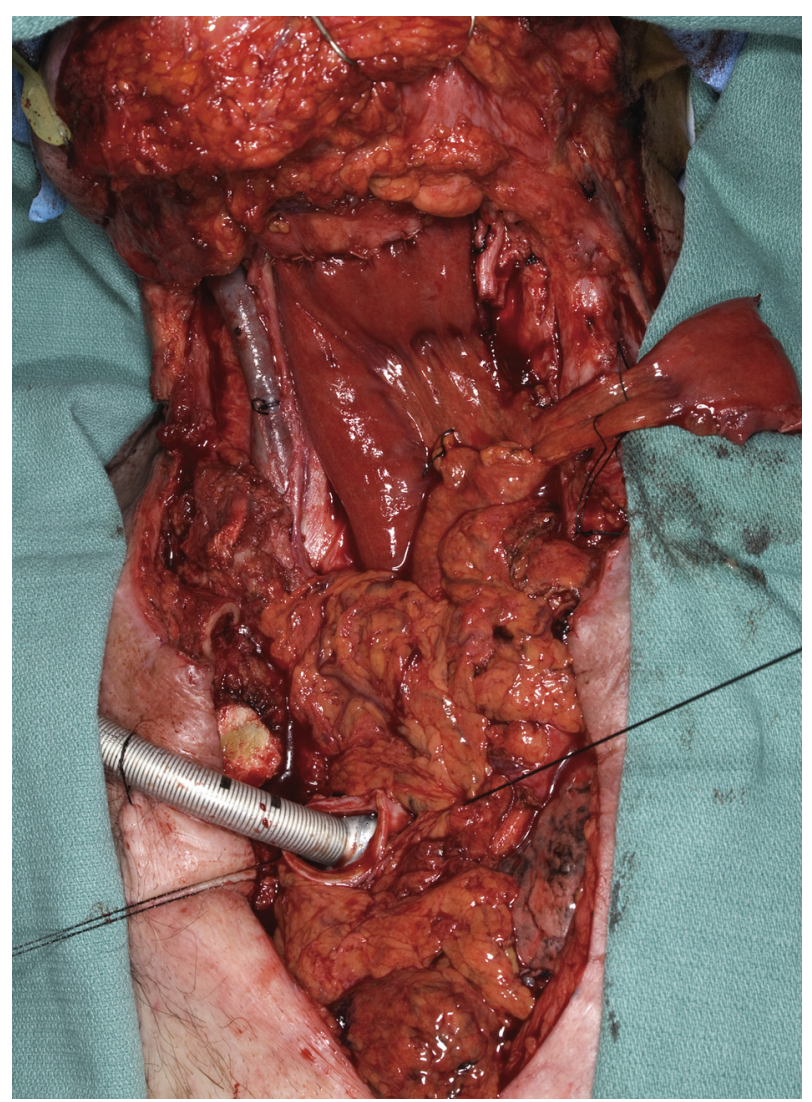

FIGURE 2. A mediastinal tracheostomy was created, and the esophagus was reconstructed with a supercharged jejunal flap. The omentum and pectoralis major muscle flap were used to cover the major vessels and tracheostomy and resurface the neck. 


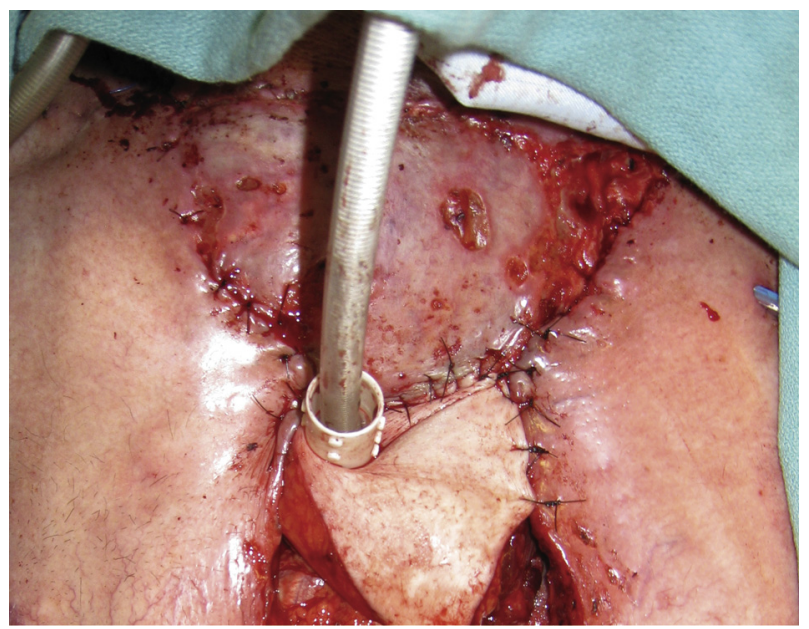

FIGURE 3. A tubed ALT free flap was used to reconstruct the trachea after debridement of the necrotic trachea.

Case 5. A bronchoesophageal fistula developed in a 53-year-old man 8 months after chemoradiation and later esophageal stent placement for a poorly differentiated squamous cell carcinoma of the middle esophagus. Tumor recurrence was confirmed. At the first stage, a right lower lobectomy with en bloc esophagectomy and mediastinal node dissection was performed with a spit fistula formation in the neck. An intercostal muscle flap was used to provide coverage of the remaining bronchial stump and pulmonary vasculature. At the second stage 6 months later, esophageal reconstruction was accomplished with a supercharged jejunal flap via the substernal route. The internal thoracic vessels served as recipient vessels to revascularize the jejunum. The patient made an uneventful recovery and was discharged on postoperative day 17 . He tolerated a regular diet

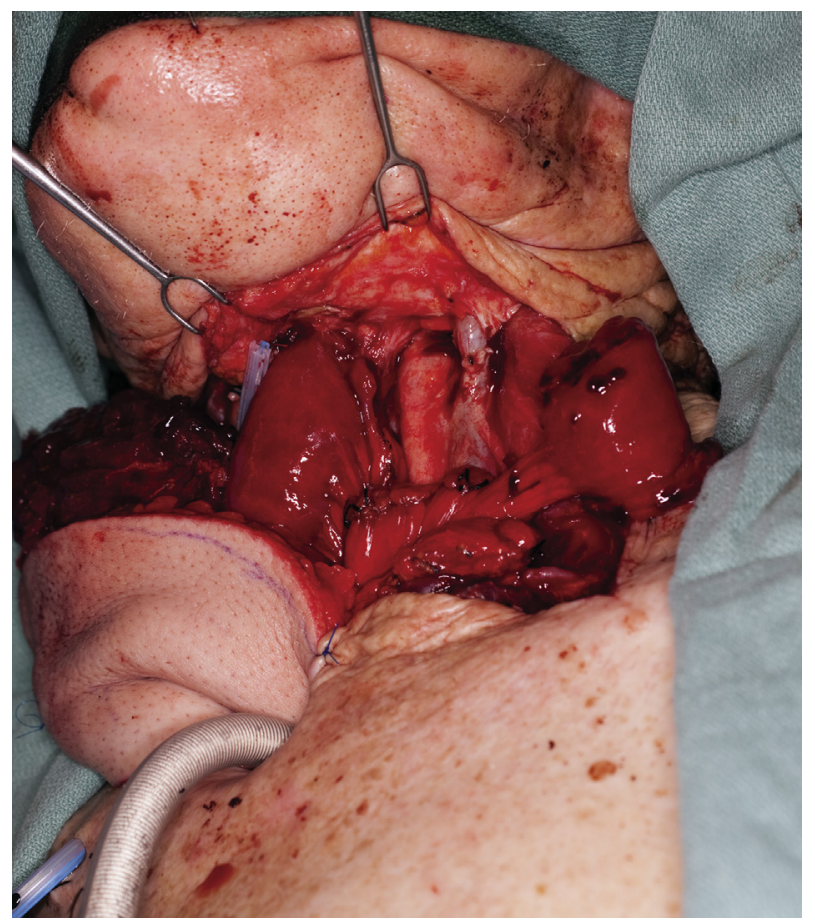

FIGURE 4. A free ALT flap was used to reconstruct the trachea and neck defect, and a supercharged jejunal flap was used to reconstruct the esophagus.

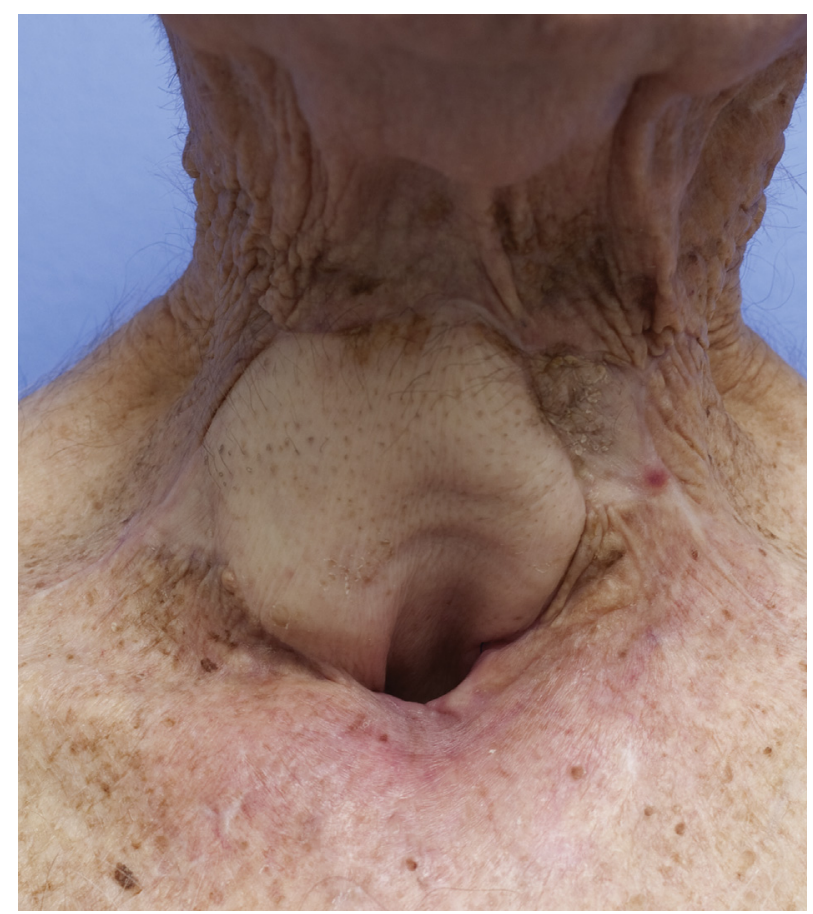

FIGURE 5. Three-month follow-up photograph shows healed ALT flap reconstruction for the tracheal defect with a stable airway.

without tube feeding and gained weight, but eventually died of recurrent disease 2 years later.

\section{DISCUSSION}

Lesions involving both the esophagus and the trachea can be life-threatening and a great challenge to surgeons. More often than not, these lesions are considered nonresectable because of the difficulties or inability to reconstruct these combined defects. In addition, patients often have had radiotherapy, greatly compromising the tissue quality and thus wound healing. Therefore, large amounts of neck and anterior chest skin defects are also common after these complex resections. In our small series of patients who have had a life-threatening tracheoesophageal (bronchoesophageal) fistula as a complication of previous cancer treatment or cancer recurrence threatening vital organs, we have demonstrated that with careful planning, lifesaving ablative surgery, and subsequent reconstruction can be performed with good functional outcomes and acceptable risk. The primary goals of treating these patients are to provide a stable airway and control infection. Simultaneous or delayed esophageal reconstruction is also desirable to maximize the patient's quality of life. This is the only series in the literature reporting the use of combined regional or free flaps with a supercharged jejunal flap for tracheal and esophageal reconstruction. Although patients with advanced cancer may eventually die of their disease, most of our patients in this series survived for more than 2 years, making such a surgical endeavor worthwhile. 
In patient 2 with tracheostomal recurrence after a previous total laryngectomy and radiotherapy, successful simultaneous tracheal and esophageal reconstruction with a free ALT flap and supercharged jejunal flap, although complex, enabled the creation of a secure airway and continuity of the alimentary tract, as well as primary wound healing with prompt recovery and return of quality of life. The paucity of double microsurgical free flap reconstructions of tracheoesophageal defects in the literature is a testament to the complexity of these procedures. The free radial forearm flap in isolation has been described for tracheostoma reconstruction after wide resection of parastomal tissues for recurrence ${ }^{10}$ and a combined free radial forearm flap and gastric pull-up used for reconstruction of the posterior tracheal wall invaded by esophageal carcinoma. ${ }^{11}$ To our knowledge, our case is the first in the literature using a free ALT flap for tracheal reconstruction and a supercharged jejunal flap transfer with microvascular anastomosis for a total esophageal reconstruction. Traditionally, such a tracheal resection with only $5 \mathrm{~cm}$ of trachea intact would mandate the creation of a mediastinal tracheostoma. Because of the need for extensive mobilization of the remaining trachea and transposition of the trachea below the great vessels, mediastinal tracheostoma is usually associated with a high morbidity and mortality, particularly as a result of innominate artery rupture. ${ }^{12-14}$ This catastrophic complication resulted from tracheostoma separation, with exposure of the great vessels, mediastinal sepsis, pressure necrosis from the trachea onto the innominate artery, or a combination thereof. Although using well-vascularized tissue to obliterate the dead space in the upper mediastinum and separate the trachea and innominate artery may decrease such risks, serious complications remain high, ${ }^{15-19}$ as exemplified in patient 1 . Severe radiation damage also may have contributed to the tracheal necrosis in this patient after the creation of a mediastinal tracheostoma. Although the trachea was subsequently reconstructed with a free ALT flap, the multidrug-resistant infection in the mediastinum and pneumonia eventually resulted in multiorgan failure and death. Lessons learned from this case prompted us to develop a strategy for simultaneous tracheoesophageal reconstruction as seen in patient 2 . Although technically demanding, such an approach eliminated the need for an extensive dissection and transposition of the remaining trachea, and provided excellent protection of the great vessels and other vital structures in the mediastinum. An alternative approach would be a 2 -stage reconstruction, which may reduce the complexity of the primary surgery. In the first stage, a secure airway can be created with the free ALT flap, which is a straightforward procedure. Esophageal continuity can be established with a supercharged jejunal flap via the substernal route in the second stage several months later, as seen in patients 3,4 , and 5 . It should be noted that soft tissue flap alone without rigid support for tracheal reconstruction is recommended only in patients who already had a total laryngectomy so that the soft tissue flap can be easily stented using a Larry tube through the stoma. Rigid support is mandatory for tracheal reconstruction with an intact larynx. ${ }^{20}$

These patients presented to us with a tracheo-(broncho-) esophageal fistula and life-threatening aspiration pneumonia with active disease. Obviously, the priority of management is to eliminate the fistula, secure the airway, and control mediastinal infection with a "damage control" lifesaving procedure. ${ }^{21}$ The esophagus is usually debrided and diverted without immediate reconstruction. A well-vascularized muscle flap is always needed to heal the tracheal or bronchial defect, obliterate the dead space, and protect vital structures. Esophagocutaneous or esophagorespiratory fistulas or fatal arterial rupture did not develop in any patient in this series. Once the patient is stabilized, fully recovered, and nutritionally improved, a second-stage esophageal reconstruction usually can be performed safely with the supercharged jejunal flap. ${ }^{6,7}$

\section{CONCLUSIONS}

Our experience suggests that complex and often life-threatening lesions involving both the trachea and the esophagus are not necessarily inoperable. With careful planning, these combined defects can be safely reconstructed with multiple flaps to secure the airway and restore alimentary tract continuity as a single-stage or 2-stage procedure with good functional outcomes and reasonable survival. Such a surgical endeavor requires close collaboration among several specialties, such as thoracic and cardiovascular surgery, head and neck surgery, plastic surgery, anesthesiology, and intensive care.

\section{Conflict of Interest Statement}

Authors have nothing to disclose with regard to commercial support.

\section{References}

1. Aly A, Jamieson GG. Reflux after oesophagectomy. Br J Surg. 2004;91:137-41. 2. DeMeester TR, Johansson KE, Franze I, Eypasch E, Lu CT, McGill JE, et al. Indications, surgical technique, and long-term functional results of colon interposition or bypass. Ann Surg. 1988;208:460-74.

3. Briel JW, Tamhankar AP, Hagen JA, DeMeester SR, Johansson J, Choustoulakis E, et al. Prevalence and risk factors for ischemia, leak, and stricture of esophageal anastomosis: gastric pull-up versus colon interposition. J Am Coll Surg. 2004;198:536-42.

4. Cense HA, Visser MR, van Sandick JW, de Boer AG, Lamme B, Obertop H, et al. Quality of life after colon interposition by necessity for esophageal cancer replacement. J Surg Oncol. 2004;88:32-8.

5. Conti M, Benhamed L, Mortuaire G, Chevalier D, Pincon C, Wurtz A. Indications and results of anterior mediastinal tracheostomy for malignancies. Ann Thorac Surg. 2010;89:1588-95.

6. Rice DC, Yu P. Use of supercharged jejunal flap for esophageal reconstruction. Op Tech Thorac Cardiovasc Surg. 2010;5:243-57. 
7. Poh M, Selber JC, Skoracki R, Walsh GL, Yu P. Technical challenges of total esophageal reconstruction using a supercharged jejunal flap. Ann Surg. 2011; 253:1122-9.

8. Yu P. Characteristics of the anterolateral thigh flap in a Western population and its application in head and neck reconstruction. Head Neck. 2004;26:759-69.

9. Yu P, Hanasono MM, Skoracki RJ, Baumann DP, Lewin JS, Weber RS, et al. Pharyngoesophageal reconstruction with the anterolateral thigh flap following total laryngopharyngectomy. Cancer. 2010;116:1718-24.

10. Cordeiro PG, Mastorakos DP, Shaha AR. The radial forearm fasciocutaneous freetissue transfer for tracheostomy reconstruction. Plast Reconstr Surg. 1996;98:354-7.

11. Nakatsuka T, Kato H, Ebihara S, Mizobuchi S, Hirano K, Harii K. Free forearm flap reconstruction of the posterior tracheal wall invaded by esophageal carcinoma. J Reconstr Microsurg. 1998;14:305-8.

12. Waddell WR, Cannon B. A technique for subtotal excision of the trachea and establishment of a sternal tracheostomy. Ann Surg. 1959;149:1-8.

13. Sisson GA, Straehley CJ Jr, Johnson NE. Mediastinal dissection for recurrent cancer after laryngectomy. Laryngoscope. 1962;72:1064-77.

14. Terz JJ, Wagman LD, King RE, Brown P, Neifeld JP, Lawrence W Jr. Results of extended resection of tumors involving the cervical part of the trachea. Surg Gynecol Obstet. 1980;151:491-6.

15. Biller HF, Krespi YP, Lawson W, Baek SM. A one-stage flap reconstruction following resection for stomal recurrence. Otolaryngol Head Neck Surg. 1980; $88: 357-60$.
16. Withers EH, Davis JL, Lynch JB. Anterior mediastinal tracheostomy with a pectoralis major musculocutaneous flap. Plast Reconstr Surg. 1981;67: 381-5.

17. Fujita H, Kakegawa T, Yamana H, Shirouzu G, Minami T. Mediastinal tracheostomy using a pectoralis major myocutaneous flap after resection of carcinoma of the esophagus involving the proximal part of the trachea. Surg Gynecol Obstet. 1990;171:403-8.

18. Granick MS, Newton ED, Dick GO. Tracheostomal reconstruction with the latissimus dorsi myocutaneous flap. Otolaryngol Head Neck Surg. 1988;99:427-9.

19. Shinoda M, Takagi I. Anterior mediastinal tracheostomy with a latissimus dorsi musculocutaneous flap. Br J Plast Surg. 1992;45:160-2.

20. Yu P, Clayman GL, Walsh GL. Long-term outcomes of microsurgical reconstruction for large tracheal defects. Cancer. 2011;117:802-8.

21. Rodriguez AN, Diaz-Jimenez JP. Malignant respiratory-digestive fistulas. Curr Opin Pulm Med. 2010;16:329-33.

Key Words: anterolateral thigh flap, esophageal cancer, esophageal reconstruction, esophagectomy, supercharged jejunal flap, trachea, tracheal reconstruction, tracheal surgery, tracheoesophageal fistula

\title{
EDITORIAL COMMENTARY
}

\section{Who is your plastic surgeon? The importance of an experienced reconstructive surgical colleague}

\author{
Thomas Ng, MD, FRCSC, FACS
}

From the Department of Surgery, The Warren Alpert Medical School of Brown University, Providence, RI.

Disclosures: Author has nothing to disclose with regard to commercial support.

Received for publication Aug 5, 2015; accepted for publication Aug 8, 2015; available ahead of print Sept 1, 2015.

Address for reprints: Thomas Ng, MD, FRCSC, FACS, 2 Dudley St, Suite 470, Providence, RI 02905 (E-mail:

tng@usasurg.org).

J Thorac Cardiovasc Surg 2015;150:1266-7

$0022-5223 / \$ 36.00$

Copyright (C) 2015 by The American Association for Thoracic Surgery

http://dx.doi.org/10.1016/j.jtcvs.2015.08.015

Tracheal and esophageal resections continue to present significant challenges to the thoracic surgeon. To date, there continues to be no reliable prosthesis, optimal reconstruction requires the use of autologous tissue, and failure of reconstruction leads to devastating consequences. Individually, tracheal and esophageal resection and reconstruction each have their own set of unique challenges, but when performed together, as described in the article in this issue of the Journal by Ghali and colleagues ${ }^{1}$ from the MD Anderson Cancer Center, the difficulty and risk of such a combined procedure undoubtedly rise exponentially.

Ghali and colleagues ${ }^{1}$ report a case series of 5 consecutive patients undergoing combined resection and reconstruction

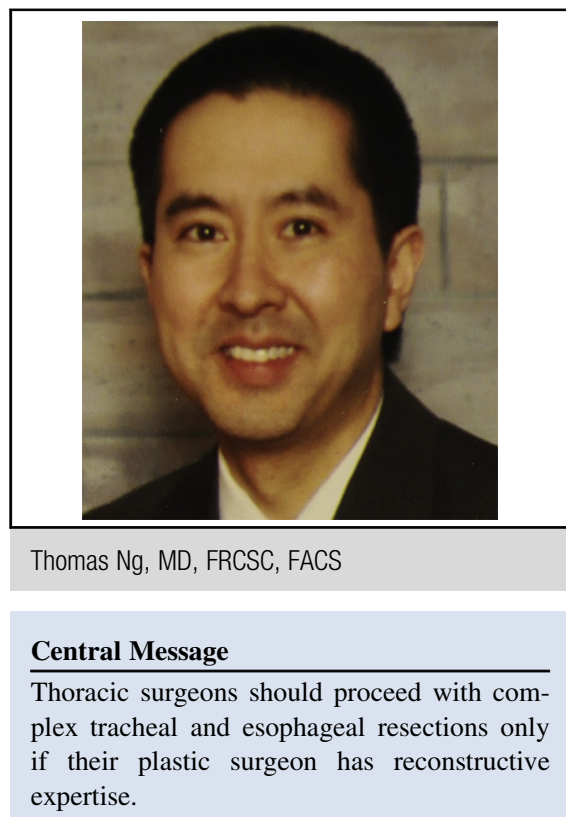

See Article page 1261. 